\title{
All Tree Amplitudes of 6D (2,0) Supergravity: Interacting Tensor Multiplets and the K3 Moduli Space
}

\author{
Matthew Heydeman, ${ }^{1}$ John H. Schwarz, ${ }^{1}$ Congkao Wen, ${ }^{2}$ and Shun-Qing Zhang ${ }^{2}$ \\ ${ }^{1}$ Walter Burke Institute for Theoretical Physics, California Institute of Technology, Pasadena, California 91125, USA \\ ${ }^{2}$ Centre for Research in String Theory, School of Physics and Astronomy, Queen Mary University of London, London, United Kingdom
}

(Received 21 December 2018; revised manuscript received 4 February 2019; published 22 March 2019)

\begin{abstract}
We present a twistorlike formula for the complete tree-level $S$ matrix of six-dimensional $(6 \mathrm{D})(2,0)$ supergravity coupled to 21 Abelian tensor multiplets. This is the low-energy effective theory that corresponds to type IIB superstring theory compactified on a K3 surface. The formula is expressed as an integral over the moduli space of certain rational maps of the punctured Riemann sphere. By studying soft limits of the formula, we are able to explore the local moduli space of this theory, $\{[S O(5,21)] /$ $[S O(5) \times S O(21)]\}$. Finally, by dimensional reduction, we also obtain a new formula for the tree-level $S$ matrix of $4 \mathrm{D} \mathcal{N}=4$ Einstein-Maxwell theory.
\end{abstract}

DOI: 10.1103/PhysRevLett.122.111604

Introduction.-To describe scattering amplitudes of supersymmetric theories in higher dimensions, Refs. [1,2] introduced a six-dimensional rational map formalism in the spirit of Refs. [3-5]. Using this formalism, extremely compact formulas were found for tree-level amplitudes of a wide range of interesting theories, including maximally supersymmetric gauge theories and supergravity in diverse dimensions, as well as the world-volume theories of probe $D$-branes and the $M 5$-brane in flat space. In the case of the M5-brane [1], which contains a chiral tensor field, the formalism circumvents a common difficulty in formulating a covariant action principle due to the self-duality constraint.

In this Letter, we continue to explore the utility of the six-dimensional (6D) rational maps and spinor-helicity formalism and present the tree-level $S$ matrix for the theory of $6 \mathrm{D}(2,0)$ supergravity. This chiral theory arises as the low-energy limit of type IIB string theory compactified on a K3 surface [6] and is particularly interesting because it describes the interaction of self-dual tensors and gravitons.

To describe massless scattering in $6 \mathrm{D}$, it is convenient to introduce spinor-helicity variables [7],

$$
p_{i}^{A B}=\lambda_{i, a}^{A} \lambda_{i, b}^{B} \epsilon^{a b}:=\left\langle\lambda_{i}^{A} \lambda_{i}^{B}\right\rangle .
$$

Here and throughout, $i=1, \ldots, n$ labels the $n$ particles, $A=1,2,3,4$ is a spinor index of the Spin $(5,1)$ Lorentz group, and $a=1,2$ is a left-handed index of the $S U(2)_{L} \times$ $S U(2)_{R}$ massless little group. This is the only nontrivial

Published by the American Physical Society under the terms of the Creative Commons Attribution 4.0 International license. Further distribution of this work must maintain attribution to the author(s) and the published article's title, journal citation, and DOI. Funded by SCOAP. little-group information that enters for chiral $(2,0)$ supersymmetry - the $(2,0)$ supergravity multiplet and a number of $(2,0)$ tensor multiplets, which contain a chiral tensor. The tensor multiplets transform as singlets of $S U(2)_{R}$, whereas the gravity multiplet is a triplet; later we will introduce the doublet index $\hat{a}$ for $S U(2)_{R}$.

We also introduce a flavor index $f_{i}$ with $i=1, \ldots, 21$ to label the 21 tensor multiplets; this is the number that arises in 6D from compactification of the NS and R fields of type IIB superstring theory on a K3 surface. It is also the unique number for which the gravitational anomalies cancel [8]. We assume that we are at generic points of the moduli space, where perturbative amplitudes are well defined [9]. Interestingly one can explore the moduli space of the theory from the $S$ matrix by studying soft limits [10]. Indeed, we derive new soft theorems from the formula we construct which describe precisely the moduli space of $6 \mathrm{D}(2,0)$ supergravity: $\{[S O(5,21)] /[S O(5) \times S O(21)]\}$.

In the rational-map formulation, amplitudes for $n$ particles are expressed as integrals over the moduli space of rational maps from the $n$-punctured Riemann sphere to the space of spinor-helicity variables. In general, the amplitudes take the following form $[1,2,11]$ :

$$
A_{n}^{6 \mathrm{D}}=\int d \mu_{n}^{6 \mathrm{D}} \mathcal{I}_{L} \mathcal{I}_{R}
$$

where $d \mu_{n}^{6 \mathrm{D}}$ is the measure encoding the $6 \mathrm{D}$ kinematics and the product $\mathcal{I}_{L} \mathcal{I}_{R}$ is the integrand that contains the dynamical information of the theories, including supersymmetry. The measure is given by

$$
d \mu_{n}^{6 \mathrm{D}}=\frac{\prod_{i=1}^{n} d \sigma_{i} \prod_{k=0}^{m} d^{8} \rho_{k}}{\operatorname{vol}\left[S L(2, \mathbb{C})_{\sigma} \times S L(2, \mathbb{C})_{\rho}\right]} \frac{1}{V_{n}^{2}} \prod_{i=1}^{n} E_{i}^{6 \mathrm{D}},
$$


and $n=2 m+2$ (we will discuss $n=2 m+1$ later). The coordinates $\sigma_{i}$ label the $n$ punctures, and $V_{n}=\prod_{i<j} \sigma_{i j}$, with $\sigma_{i j}=\sigma_{i}-\sigma_{j}$. They are determined up to an overall $S L(2, \mathbb{C})_{\sigma}$ Möbius group transformation, whose "volume" is divided out in a standard way. The 6D scattering equations are given by

$$
E_{i}^{6 \mathrm{D}}=\delta^{6}\left(p_{i}^{A B}-\frac{\left\langle\rho^{A}\left(\sigma_{i}\right) \rho^{B}\left(\sigma_{i}\right)\right\rangle}{\prod_{j \neq i} \sigma_{i j}}\right) .
$$

These maps are given by degree- $m$ polynomials $\rho_{a}^{A}(\sigma)=$ $\sum_{k=0}^{m} \rho_{a, k}^{A} \sigma^{k}$, which are determined up to an overall $S L(2, \mathbb{C})_{\rho}$ transformation, whose volume is divided out. This group is a complexification of $S U(2)_{L}$.

It is straightforward to see that Eq. (4) implies the onshell conditions $p_{i}^{2}=0$ and momentum conservation. Furthermore, as shown in Refs. [1,2], this construction implies that the integrals are completely localized on the $(n-3)$ ! solutions, which are equivalent to those of the general-dimensional scattering equations [11],

$$
\sum_{i \neq j} \frac{p_{i} \cdot p_{j}}{\sigma_{i j}}=0 \text { for all } j .
$$

We will see shortly that, unlike the general-dimensional scattering equations, the use of the spinor-helicity coordinates and 6D scattering equations allows us to make supersymmetry manifest.

Now consider $n=2 m+1$, for which we have [2]

$d \mu_{n}^{6 \mathrm{D}}=\frac{\left(\prod_{i=1}^{n} d \sigma_{i} \prod_{k=0}^{m-1} d^{8} \rho_{k}\right) d^{4} \omega\langle\xi d \xi\rangle}{\operatorname{vol}\left[S L(2, \mathbb{C})_{\sigma}, S L(2, \mathbb{C})_{\rho}, T\right]} \frac{1}{V_{n}^{2}} \prod_{i=1}^{n} E_{i}^{6 \mathrm{D}}$.

The polynomials $\rho_{a}^{A}(\sigma)$ now are given by

$$
\rho_{a}^{A}(\sigma)=\sum_{k=0}^{m-1} \rho_{a, k}^{A} \sigma^{k}+\omega^{A} \xi_{a} \sigma^{m},
$$

and there is a shift symmetry $T(\alpha)$ acting on $\omega^{A}$ : $\omega^{A} \rightarrow \omega^{A}+\alpha\left\langle\xi \rho_{m-1}^{A}\right\rangle$, which we also have to mod out.

Here we review the integrand factors for $6 \mathrm{D}(2,2)$ supergravity since they will be relevant. For $(2,2)$ supergravity, we have

$$
\mathcal{I}_{L}=\operatorname{det}^{\prime} S_{n}, \quad \mathcal{I}_{R}=\Omega_{F}^{(2,2)},
$$

where $S_{n}$ is an $n \times n$ antisymmetric matrix with entries $\left[S_{n}\right]_{i j}=\left(p_{i} \cdot p_{j} / \sigma_{i j}\right)$. This matrix has rank $(n-2)$, and the reduced Pfaffian and determinant are defined as

$$
\operatorname{Pf}^{\prime} S_{n}=\frac{(-1)^{i+j}}{\sigma_{i j}} \operatorname{Pf} S_{i j}^{i j}, \quad \operatorname{det}^{\prime} S_{n}=\left(\mathrm{Pf}^{\prime} S_{n}\right)^{2} .
$$

Here $S_{i j}^{i j}$ means that the $i$ th and $j$ th rows and columns of $S_{n}$ are removed, and the result is $i, j$ independent [12]. $\Omega_{F}^{(2,2)}$ is a fermionic function of Grassmann coordinates $\eta_{i}^{I a}, \tilde{\eta}_{i}^{\hat{I}} \hat{a}$, which we use to package the supermultiplet of on-shell states into a "superfield,"

$$
\begin{aligned}
\Phi^{(2,2)}(\eta, \tilde{\eta})= & \phi^{\prime}+\cdots+\eta_{a}^{I} \eta_{I, b} B^{a b}+\tilde{\eta}^{\hat{I}}, \hat{a} \tilde{\eta}_{\hat{l}}^{\hat{b}} B_{\hat{a} \hat{b}}+\cdots \\
& +\eta_{a}^{I} \eta_{I, b} \tilde{\eta}_{\hat{a}}^{\hat{I}} \tilde{\eta}_{\hat{\eta}, \hat{b}} G^{a b \hat{a} \hat{b}}+\cdots+(\eta)^{4}(\tilde{\eta})^{4} \bar{\phi}^{\prime},
\end{aligned}
$$

where $B^{a b}$ and $B_{\hat{a} \hat{b}}$ are self-dual and anti-self-dual twoforms, and $G^{a b \hat{a} \hat{b}}$ is the graviton. Here $I, \hat{I}=1,2$ are the $R$-symmetry indices corresponding to an $S U(2) \times S U(2)$ subgroup of the full $U S p(4) \times U S p(4) R$ symmetry. The fermionic function $\Omega_{F}^{(2,2)}$ imposes the conservation of supercharge, which may be viewed as a double copy, $\Omega_{F}^{(2,2)}=\Omega_{F}^{(2,0)} \Omega_{F}^{(0,2)}$, and $\Omega_{F}^{(2,0)}$ is given by

$$
\Omega_{F}^{(2,0)}=V_{n} \prod_{k=0}^{m} \delta^{4}\left(\sum_{i=1}^{n} C_{a, k ; i, b} \eta_{i}^{I b}\right) .
$$

The $n \times 2 n$ matrices $C_{a, k i, b}=\left(W_{i}\right)_{a}^{b} \sigma_{i}^{k}$ and $\left(W_{i}\right)_{a}^{b}$ can be expressed in terms of $\rho_{a}^{A}\left(\sigma_{i}\right)$ via

$$
p_{i}^{A B} W_{i, b}^{a}=\frac{\rho^{[A, a}\left(\sigma_{i}\right) \lambda_{i, b}^{B]}}{\prod_{j \neq i} \sigma_{i j}},
$$

which is independent of $A, B$, and satisfies $\operatorname{det} W_{i}=$ $\prod_{j \neq i} \sigma_{i j}^{-1}$. The matrix $C_{a, k i, b}$ is a symplectic Grassmannian which was used in Ref. [2] as an alternative way to impose the $6 \mathrm{D}$ scattering equations. $\Omega_{F}^{(0,2)}$ is the conjugate of $\Omega_{F}^{(2,0)}$, and the definition is identical, with the understanding that we use the right-handed variables, such as $\tilde{\eta}_{\hat{a}}^{\hat{I}}, \tilde{\lambda}_{\hat{A} \hat{a}}, \tilde{\rho}_{\hat{A} \hat{a}}, \tilde{\xi}_{\hat{a}}$, $\left(\tilde{W}_{i}\right)_{\hat{a}}^{\hat{b}}$, etc.

For $n=2 m+1$, the integrands take a slightly different form. For the fermionic part, we have

$$
\begin{aligned}
\Omega_{F}^{(2,0)}= & V_{n} \prod_{k=0}^{m-1} \delta^{4}\left(\sum_{i=1}^{n} C_{a, k ; i, b} \eta_{i}^{I b}\right) \\
& \times \delta^{2}\left(\sum_{i=1}^{n} \xi^{a} C_{a, m ; i, b} \eta_{i}^{I b}\right)
\end{aligned}
$$

whereas the $n \times n$ matrix $S_{n}$ is modified to an $(n+1) \times$ $(n+1)$ matrix, which we denote $\hat{S}_{n} . \hat{S}_{n}$ is defined in the same way as $S_{n}$, but with $i, j=1, \ldots, n, \star$. Here $\sigma_{\star}$ is a reference puncture, and $p_{\star}$ is given by

$$
p_{\star}^{A B}=\frac{2 q^{[A} p^{B] C}\left(\sigma_{\star}\right) \tilde{q}_{C}}{q^{D}\left[\tilde{\rho}_{D}\left(\sigma_{\star}\right) \tilde{\xi}\right]\left\langle\rho^{E}\left(\sigma_{\star}\right) \xi\right\rangle \tilde{q}_{E}},
$$

where $q$ and $\tilde{q}$ are arbitrary spinors.

$6 D(2,0)$ supergravity.-The $6 \mathrm{D}(2,0)$ supergravity theory contains 21 tensor multiplets and the graviton 
multiplet. The superfield of the tensor multiplet is a singlet of the little group,

$$
\Phi(\eta)=\phi+\cdots+\eta_{a}^{I} \eta_{I, b} B^{a b}+\cdots+(\eta)^{4} \bar{\phi}
$$

where $a, b=1,2$ are the $S U(2)_{L}$ little-group indices. The graviton multiplet transforms as a $(\mathbf{1 , 3})$ of the little group, so the superfield carries explicit $S U(2)_{R}$ indices,

$\Phi_{\hat{a} \hat{b}}(\eta)=B_{\hat{a} \hat{b}}+\cdots+\eta_{a}^{I} \eta_{I, b} G_{\hat{a} \hat{b}}^{a b}+\cdots+(\eta)^{4} \bar{B}_{\hat{a} \hat{b}}$,

and $\Phi_{\hat{a} \hat{b}}(\eta)=\Phi_{\hat{b} \hat{a}}(\eta)$. We see that both the tensor multiplet and the graviton multiplet can be obtained from the $6 \mathrm{D}$ $(2,2)$ superfield in Eq. (10) via supersymmetry (SUSY) reductions $[13,14]$,

$$
\begin{aligned}
\Phi(\eta) & =\left.\int d \tilde{\eta}_{\hat{a}}^{\hat{I}} d \tilde{\eta}_{\hat{I}}^{\hat{a}} \Phi^{(2,2)}(\eta, \tilde{\eta})\right|_{\tilde{\eta} \rightarrow 0}, \\
\Phi_{\hat{a} \hat{b}}(\eta) & =\left.\int d \tilde{\eta}_{\hat{a}}^{\hat{I}} d \tilde{\eta}_{\hat{I} \hat{b}} \Phi^{(2,2)}(\eta, \tilde{\eta})\right|_{\tilde{\eta} \rightarrow 0} .
\end{aligned}
$$

These integrals have the effect of projecting onto the right-handed $U S p(4) R$-symmetry singlet sector, which reduces $(2,2) \rightarrow(2,0)$. Using the reduction, the amplitudes of $(2,0)$ supergravity with $n_{1}$ supergravity multiplets and $n_{2}$ tensor multiplets of the same flavor $\left(n_{1}+n_{2}=n\right)$ can be obtained from the $(2,2)$ supergravity amplitude via

$$
A_{n_{1}, n_{2}}^{(2,0)}=\int \prod_{i \in n_{1}} d \tilde{\eta}_{i, \hat{a}_{i}}^{\hat{I}} d \tilde{\eta}_{i, \hat{b} \hat{b}_{i}} \prod_{j \in n_{2}} d \tilde{\eta}_{j, \hat{a}_{j}}^{\hat{\jmath}} d \tilde{\eta}_{j, \hat{J}}^{\hat{a}_{j}} A_{n}^{(2,2)}(\eta, \tilde{\eta}) .
$$

Note that $A_{n}^{(2,2)}(\eta, \tilde{\eta}) \sim \eta^{2 n} \tilde{\eta}^{2 n}$, so the integration removes all $\tilde{\eta}$ 's. The fermionic integration can be performed using Eqs. (8) and (11) [or Eq. (13) for odd $n$ ], and we obtain

$$
A_{n_{1}, n_{2}}^{(2,0)}=\int d \mu_{n}^{6 \mathrm{D}} \tilde{M}_{\hat{a} \hat{b}}^{n_{1} n_{2}} V_{n} \operatorname{det}^{\prime} S_{n} \Omega_{F}^{(2,0)},
$$

where $\tilde{M}_{\hat{a} \hat{b}}^{n_{1} n_{2}}$, which we will define shortly, is obtained by integrating out $\Omega_{F}^{(0,2)}$.

We begin with $n$ even, as the odd- $n$ case works in a similar fashion. Introducing the $n \times n$ matrix

$$
\tilde{M}_{\hat{a}_{1} \cdots \hat{a}_{n}}=\left(\begin{array}{cccc}
\tilde{C}_{\hat{1}, 0 ; 1, \hat{a}_{1}} & \tilde{C}_{\hat{1}, 0 ; 2, \hat{a}_{2}} & \cdots & \tilde{C}_{\hat{1}, 0 ; n, \hat{a}_{n}} \\
\vdots & \vdots & \cdots & \vdots \\
\tilde{C}_{\hat{1}, m ; 1, \hat{a}_{1}} & \tilde{C}_{\hat{1}, m ; 2, \hat{a}_{2}} & \cdots & \tilde{C}_{\hat{1}, m ; n, \hat{a}_{n}} \\
\tilde{C}_{\hat{2}, 0 ; 1, \hat{a}_{1}} & \tilde{C}_{\hat{2}, 0 ; 2, \hat{a}_{2}} & \cdots & \tilde{C}_{\hat{2}, 0 ; n, \hat{a}_{n}} \\
\vdots & \vdots & \cdots & \vdots \\
\tilde{C}_{\hat{2}, m ; 1, \hat{a}_{1}} & \tilde{C}_{\hat{2}, m ; 2, \hat{a}_{2}} & \cdots & \tilde{C}_{\hat{2}, m ; n, \hat{a}_{n}}
\end{array}\right),
$$

$\tilde{M}_{\hat{a} \hat{b}}^{n_{1} n_{2}}$ is then given by

$$
\tilde{M}_{\hat{a} \hat{b}}^{n_{1} n_{2}}=\operatorname{det} \tilde{M}_{\hat{a}_{1} \cdots \hat{a}_{n}} \operatorname{det} \tilde{M}_{\hat{b}_{1} \cdots \hat{b}_{n}} .
$$

Note that here $\hat{a}$ and $\hat{b}$ denote sets of indices. The indices $\hat{a}_{i}, \hat{b}_{i}$ are contracted if $i \in n_{2}$, whereas for $j \in n_{1}$ we symmetrize $\hat{a}_{j}, \hat{b}_{j}$. This corresponds to constructing littlegroup singlets for tensor multiplets and triplets for graviton multiplets. After the contraction and symmetrization, the result of (20) simplifies drastically [15],

$$
\tilde{M}_{\hat{a} \hat{b}}^{n_{1} n_{2}} \rightarrow \frac{\operatorname{Pf} X_{n_{2}}}{V_{n_{2}}} \tilde{M}_{\hat{a} \hat{b}}^{n_{1} 0},
$$

where $X_{n_{2}}$ is an $n_{2} \times n_{2}$ antisymmetric matrix given by

$$
\left[X_{n_{2}}\right]_{i j}= \begin{cases}\frac{1}{\sigma_{i j}} & \text { if } i \neq j, \\ 0 & \text { if } i=j,\end{cases}
$$

and $\tilde{M}_{\hat{a} \hat{b}}^{n_{1} 0}$ contains only the graviton multiplets. Let us remark that the simplification (21) (especially the appearance of $\operatorname{Pf} X_{n_{2}}$ ) will be crucial for the generalization to amplitudes with multiple tensor flavors which is more interesting and relevant for type IIB on K3.

At this point in the analysis, we have obtained the treelevel amplitudes of 6D $(2,0)$ supergravity with a single flavor of tensor multiplets:

$$
A_{n_{1}, n_{2}}^{(2,0)}=\int d \mu_{n}^{6 \mathrm{D}} \frac{\operatorname{Pf} X_{n_{2}}}{V_{n_{2}}} \tilde{M}_{\hat{a} \hat{b}}^{n_{1} 0} V_{n} \operatorname{det}^{\prime} S_{n} \Omega_{F}^{(2,0)} .
$$

The factor $\operatorname{Pf} X_{n_{2}}$ requires the nonvanishing amplitudes to contain an even number $n_{2}$ of tensor multiplets, as expected. For odd $n$, the matrix $\tilde{M}_{\hat{a}_{1} \cdots \hat{a}_{n}}$ is given by

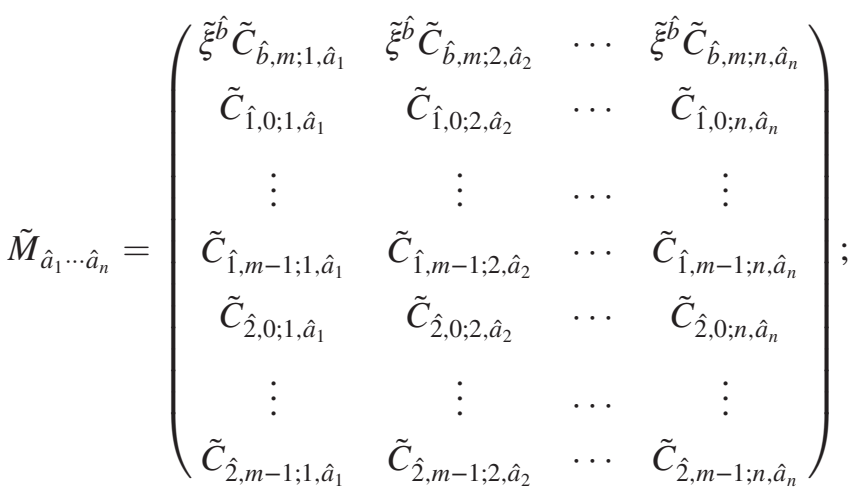

recall that $\tilde{\xi}^{\hat{b}}$ is the right-hand version of $\xi^{b}$ in Eq. (7). Then the amplitudes take the same form,

$$
A_{n_{1}, n_{2}}^{(2,0)}=\int d \mu_{n}^{6 \mathrm{D}} \frac{\operatorname{Pf} X_{n_{2}}}{V_{n_{2}}} \tilde{M}_{\hat{a} \hat{b}}^{n_{1} 0} V_{n} \operatorname{det}^{\prime} \hat{S}_{n} \Omega_{F}^{(2,0)} .
$$

Multiflavor tensor multiplets: As we have emphasized, identity (21) is crucial for the generalization to multiple 
tensor flavors, which is required for the 6D $(2,0)$ supergravity. Indeed, the formula takes a form similar to that of an Einstein-Maxwell theory worked out by Cachazo et al. [12], especially the object $\operatorname{Pf} X_{n_{2}}$. In that case, in passing from single- $U(1)$ photons to multiple- $U(1)$ ones, one simply replaced the matrix $X_{n}$ with $\mathcal{X}_{n}$ [12],

$$
\left[\mathcal{X}_{n}\right]_{i j}= \begin{cases}\frac{\delta_{f_{i} f_{j}}}{\sigma_{i j}} & \text { if } i \neq j \\ 0 & \text { if } i=j\end{cases}
$$

which allows the introduction of multiple distinct flavors: namely, $f_{i}, f_{j}$ are flavor indices, and $\delta_{f_{i} f_{j}}=1$ if particles $i$, $j$ are of the same flavor; otherwise $\delta_{f_{i} f_{j}}=0$. Inspired by this result, we are led to a proposal for the complete treelevel $S$ matrix of $6 \mathrm{D}(2,0)$ supergravity with multiple flavors of tensor multiplets:

$$
A_{n_{1}, n_{2}}^{(2,0)}=\int d \mu_{n}^{6 \mathrm{D}} \frac{\operatorname{Pf}_{\mathcal{X}_{n_{2}}}}{V_{n_{2}}} \tilde{M}_{\hat{a} \hat{b}}^{n_{1} 0} V_{n} \operatorname{det}^{\prime} S_{n} \Omega_{F}^{(2,0)} .
$$

Again, the 6D scattering equations and integrands take different forms depending on whether $n$ is even or odd [16]. Since $n_{2}$ is necessarily even, this is equivalent to distinguishing whether $n_{1}$ is even or odd.

Equation (26) is our main result, which is a localized integral formula that describes all tree-level superamplitudes of Abelian tensor multiplets (with multiple flavors) coupled to gravity multiplets. We can verify that it has all of the correct properties. For instance, due to the fact that all of the building blocks of the formula come from either $6 \mathrm{D}$ $(2,2)$ supergravity or Einstein-Maxwell theory, they all behave properly in the factorization limits and transform correctly under the symmetries: $S L(2, \mathbb{C})_{\sigma}, S L(2, \mathbb{C})_{\rho}$, etc. Also, as we will show later, when reduced to $4 \mathrm{D}$, the proposed formula produces (supersymmetric) EinsteinMaxwell amplitudes, which is another consistency check. Finally, it is straightforward to check that the formula gives correct low-point amplitudes, e.g., [17],

$$
\begin{aligned}
& A_{0,4}^{(2,0)}=\delta^{8}(Q)\left(\frac{\delta^{f_{1} f_{2}} \delta^{f_{3} f_{4}}}{s_{12}}+\frac{\delta^{f_{1} f_{3}} \delta^{f_{2} f_{4}}}{s_{13}}+\frac{\delta^{f_{2} f_{3}} \delta^{f_{1} f_{4}}}{s_{23}}\right), \\
& A_{2,2}^{(2,0)}=\delta^{f_{1} f_{2}} \frac{\delta^{8}(Q)\left[1_{\hat{a}_{1}} 2_{\hat{a}_{2}} 3_{\hat{a}_{3}} 4_{\hat{a}_{4}}\right]\left[1^{\hat{a}_{1}} 2^{\hat{a}_{2}} 3_{\hat{b}_{3}}{ }_{\hat{b}_{4}}\right]}{s_{12} s_{23} s_{31}}+\text { sym. }
\end{aligned}
$$

We symmetrize $\hat{a}_{3}, \hat{b}_{3}$ and $\hat{a}_{4}, \hat{b}_{4}$ for the graviton multiplets, $\left[1_{\hat{a}_{1}} 2_{\hat{a}_{2}} 3_{\hat{a}_{3}} 4_{\hat{a}_{4}}\right]=\epsilon_{A B C D} \tilde{\lambda}_{1 \hat{a}_{1}}^{A} \tilde{\lambda}_{2 \hat{a}_{2}}^{B} \tilde{\lambda}_{3 \hat{a}_{3}}^{C} \tilde{\lambda}_{4 \hat{a}_{4}}^{D}$, and $\delta^{8}(Q)=$ $\delta^{8}\left(\sum_{i=1}^{4} \lambda_{i, a}^{A} \eta_{i}^{I a}\right)$.

The K3 moduli space from soft limits.-Type IIB string theory compactified on K3 has a well studied moduli space described by the coset [18],

$$
\mathcal{M}_{(2,0)}=S O(5,21 ; \mathbb{Z}) \backslash S O(5,21) /[S O(5) \times S O(21)] .
$$

The discrete group is invisible in the supergravity approximation, so we concern ourselves with the local form of the moduli space of supergravity theory-namely, $\{[S O(5,21)] /[S O(5) \times S O(21)]\}$. It has a dimension of 105 , which corresponds precisely to the 105 scalars in the 21 tensor multiplets. These scalars are Goldstone bosons of the breaking of $S O(5,21)$ to $S O(5) \times S O(21)$, which are the $R$ symmetry and the flavor symmetry, respectively. Therefore, the scalars obey soft theorems, which are the tools to explore the structure of the moduli space directly from the $S$ matrix [10].

We find that the amplitudes behave like pion amplitudes with "Adler's zero" [19] in the single-soft limit. Indeed, for $p_{1} \rightarrow 0$, we find that

$$
A_{n}\left(\phi_{1}^{f_{1}}, 2, \ldots, n\right) \rightarrow O\left(p_{1}\right),
$$

and the same for other scalars in the tensor multiplets. The commutator algebra of the coset space may be explored by considering double-soft limits for scalars. Beginning with the flavor symmetry, we find for $p_{1}$, $p_{2} \rightarrow 0$ simultaneously

$A_{n}\left(\phi_{1}^{f_{1}}, \bar{\phi}_{2}^{f_{2}}, \ldots\right) \rightarrow \frac{1}{2} \sum_{i=3}^{n} \frac{p_{i} \cdot\left(p_{1}-p_{2}\right)}{p_{i} \cdot\left(p_{1}+p_{2}\right)} R_{i}^{f_{1} f_{2}} A_{n-2}$,

where the $f_{i}$ 's are flavor indices and $R_{i}^{f_{1} f_{2}}$ is a generator of the unbroken $S O(21)$, which may be viewed as the result of the commutator of two broken generators. $R_{i}^{f_{1} f_{2}}$ acts on superfields as

$$
\begin{aligned}
& R_{i}^{f_{1} f_{2}} \Phi_{i}^{f_{2}}=\Phi_{i}^{f_{1}}, \quad R_{i}^{f_{1} f_{2}} \Phi_{i}^{f_{1}}=-\Phi_{i}^{f_{2}}, \\
& R_{i}^{f_{1} f_{2}} \Phi_{i}^{f_{3}}=0, \quad R_{i}^{f_{1} f_{2}} \Phi_{i, \hat{a} \hat{b}}=0,
\end{aligned}
$$

where $f_{3} \neq f_{1}, f_{2}$. Therefore, the generator exchanges tensor multiplets of flavor $f_{1}$ with ones of $f_{2}$ and sends all others and the graviton multiplet to 0 .

To study the $S O(5) R$-symmetry generators, we take soft limits of two scalars which do not form an $R$-symmetry singlet. For instance,

$$
A_{n}\left(\bar{\phi}_{1}, \phi_{2}^{I J}, \ldots\right) \rightarrow \frac{1}{2} \sum_{i=3}^{n} \frac{p_{i} \cdot\left(p_{1}-p_{2}\right)}{p_{i} \cdot\left(p_{1}+p_{2}\right)} R_{i}^{I J} A_{n-2},
$$

with $R_{i}^{I J}=\eta_{i, a}^{I} \eta_{i}^{J, a}$. Similarly, other choices of soft scalars lead to the remaining $R$-symmetry generators:

$$
R_{i, I J}=\frac{\partial}{\partial \eta_{i, a}^{I}} \frac{\partial}{\partial \eta_{i}^{J, a}}, \quad R_{i, J}^{I}=\eta_{i, a}^{I} \frac{\partial}{\partial \eta_{i, a}^{J}} .
$$

Finally, we consider the cases in which soft scalars carry different flavors and do not form an $R$-symmetry singlet. This actually leads to new soft theorems, 
$A_{n}\left(\bar{\phi}_{1}^{f_{1}}, \phi_{2}^{f_{2}, I J}, \ldots\right) \rightarrow \sum_{i=3}^{n} \frac{p_{1} \cdot p_{2}}{p_{i} \cdot\left(p_{1}+p_{2}\right)} R_{i}^{f_{1} f_{2}} R_{i}^{I J} A_{n-2}$,

and one may proceed similarly for other $R$-symmetry generators. The results of the soft limits now contain both flavor and $R$-symmetry generators, reflecting the direct product structure in $\{[S O(5,21)] /[S O(5) \times S O(21)]\}$. This is a new phenomenon that is not present in pure $(2,0)$ supergravity $[10,20]$.

The above soft theorems may be obtained by analyzing how the integrand and the scattering equations behave in the limits. For instance, the vanishing of the amplitudes in the single-soft limits is due to

$$
\int d \mu_{n}^{6 D} \sim O\left(p_{1}^{-1}\right), \quad \operatorname{det}^{\prime} S_{n} \sim O\left(p_{1}^{2}\right),
$$

and the rest remains finite. The double-soft theorems require more careful analysis along the lines of, e.g., Ref. [21]. The structures of double-soft theorems, however, are already indicated by knowing the four-point amplitudes, since important contributions are diagrams with a four-point amplitude on one side such that the propagator becomes singular in the limit [22]. Finally, we have also checked the soft theorems explicitly using formula (26) for various examples.

$4 D \mathcal{N}=4$ Einstein-Maxwell theory.-One can dimensionally reduce $6 \mathrm{D}(2,0)$ supergravity to obtain $4 \mathrm{D} \mathcal{N}=4$ Einstein-Maxwell theory. The tree-level amplitudes of this theory capture the leading low-energy behavior of type IIB (or type IIA) superstring theory on $\mathrm{K} 3 \times \mathrm{T}^{2}$.

The reduction to $4 \mathrm{D}$ can be obtained by decomposing the 6D spinor as $A \rightarrow \alpha=1,2, \dot{\alpha}=3,4$. The compact momenta are $P_{i}^{\alpha \beta}=P_{i}^{\dot{\alpha} \dot{\beta}}=0$; this is implemented by $\lambda_{a}^{A} \rightarrow \lambda_{+}^{\alpha}=0$ and $\lambda_{-}^{\dot{\alpha}}=0$.

The $6 \mathrm{D}$ tensor superfield becomes an $\mathcal{N}=4$ vector multiplet in $4 \mathrm{D}$, in a nonchiral form [1,23],

$$
\begin{aligned}
\Phi\left(\eta_{a}\right) \rightarrow & V_{\mathcal{N}=4}\left(\eta_{+}, \eta_{-}\right)=\phi+\eta_{-}^{\hat{I}} \psi_{\hat{I}}^{-}+\cdots \\
& +\left(\eta_{+}\right)^{2} A^{+}+\left(\eta_{-}\right)^{2} A^{-}+\cdots+\left(\eta_{+}\right)^{2}\left(\eta_{-}\right)^{2} \bar{\phi} .
\end{aligned}
$$

Dimensional reduction of $\Phi^{\hat{a} \hat{b}}(\eta)$ is analogous. It separates into three cases, where $\Phi^{\hat{+} \hat{-}} \rightarrow V_{\mathcal{N}=4}\left(\eta_{+}, \eta_{-}\right)$, and $\Phi^{\hat{+} \hat{+}}, \Phi^{\hat{A} \hat{A}}$ become a pair of positive- and negative-helicity graviton multiplets

$$
\begin{aligned}
\Phi^{\hat{+} \hat{+}}\left(\eta_{a}\right) \rightarrow & \mathcal{G}_{\mathcal{N}=4}^{+}\left(\eta_{+}, \eta_{-}\right)=A^{+}+\eta_{-}^{\hat{I}} \psi_{\hat{I}}^{-+}+\cdots \\
& +\left(\eta_{+}\right)^{2} G^{++}+\left(\eta_{-}\right)^{2} \phi+\cdots+\left(\eta_{+}\right)^{2}\left(\eta_{-}\right)^{2} \bar{A}^{+},
\end{aligned}
$$

$$
\begin{aligned}
\Phi^{\hat{\alpha}}\left(\eta_{a}\right) \rightarrow & \mathcal{G}_{\mathcal{N}=4}^{-}\left(\eta_{+}, \eta_{-}\right)=\bar{A}^{-}+\eta_{-}^{\hat{I}} \Psi_{\hat{I}}^{--}+\cdots \\
& +\left(\eta_{-}\right)^{2} G^{--}+\left(\eta_{+}\right)^{2} \bar{\phi}+\cdots+\left(\eta_{+}\right)^{2}\left(\eta_{-}\right)^{2} A^{-} .
\end{aligned}
$$

We see that the on-shell spectrum of the 4D supergravity theory consists of the $\mathcal{G}^{+}$and $\mathcal{G}^{-}$superfields coupled to 22 $\mathcal{N}=4$ Maxwell multiplets.

We are now ready to perform the dimensional reduction on Eq. (26) [24]. First, the 6D measure reduces to

$$
d \mu^{4 \mathrm{D}}=\frac{\prod_{i=1}^{n} d \sigma_{i} \prod_{k=0}^{d} d^{2} \rho_{k} \prod_{k=0}^{\tilde{d}} d^{2} \tilde{\rho}_{k}}{\operatorname{vol}\left(S L(2, \mathbb{C})_{\sigma} \times G L(1, \mathbb{C})\right)} \frac{1}{R(\rho) R(\tilde{\rho})} \prod_{i=1}^{n} E_{i}^{4 \mathrm{D}}
$$

where $R(\rho), R(\tilde{\rho})$ are the resultants of the polynomials

$$
\rho^{\alpha}(\sigma)=\sum_{k=0}^{d} \rho_{k}^{\alpha} \sigma^{k}, \quad \tilde{\rho}^{\dot{\alpha}}(\sigma)=\sum_{k=0}^{\tilde{d}} \tilde{\rho}_{k}^{\dot{\alpha}} \sigma^{k},
$$

with $d+\tilde{d}=n-2$, and the 4D scattering equations are given by

$$
E_{i}^{4 \mathrm{D}}=\delta^{4}\left(p_{i}^{\alpha \dot{\alpha}}-\frac{\rho^{\alpha}\left(\sigma_{i}\right) \tilde{\rho}^{\dot{\alpha}}\left(\sigma_{i}\right)}{\prod_{j \neq i} \sigma_{i j}}\right) .
$$

The $2 \times 2$ matrix $\left(\tilde{W}_{i}\right)_{a b}$ reduces to

$$
\left(\tilde{W}_{i}\right)_{\hat{+} \hat{+}}=\left(\tilde{W}_{i}\right)_{\hat{\wedge} \hat{\imath}}=0, \quad\left(\tilde{W}_{i}\right)_{\hat{+} \hat{\imath}}=t_{i}, \quad\left(\tilde{W}_{i}\right)_{\hat{\imath} \hat{+}}=\tilde{t}_{i},
$$

with $t_{i}=\left[\lambda_{i}^{\alpha} / \rho^{\alpha}\left(\sigma_{i}\right)\right], \tilde{t}_{i}=\left[\tilde{\lambda}_{i}^{\dot{\alpha}} / \tilde{\rho}^{\dot{\alpha}}\left(\sigma_{i}\right)\right]$ (independent of $\alpha$, $\dot{\alpha})$, and $t_{i} \tilde{t}_{i}=\prod_{j \neq i}\left(1 / \sigma_{i j}\right)$. As for the integrand, the parts that reduce to $4 \mathrm{D}$ nontrivially are

$$
\tilde{M}_{\hat{a} \hat{b}}^{n_{1}} \rightarrow \tilde{T}_{\hat{a} \hat{b}}^{n_{1}}, \quad \operatorname{det}^{\prime} S_{n} \rightarrow R^{2}(\rho) R^{2}(\tilde{\rho}) V_{n}^{-2} .
$$

Assuming that we have $m_{1} \mathcal{G}^{+}$superparticles and $m_{2} \mathcal{G}^{-}$, with $m_{1}+m_{2}=n_{1}$ [25], we find that $\tilde{T}^{n_{1}}$ is given by

$$
\tilde{T}^{n_{1}}=T_{+}^{m_{1}} T_{-}^{m_{2}}=\left(V_{m_{1}}^{2} \prod_{i \in m_{1}} t_{i}^{2}\right)\left(V_{m_{2}}^{2} \prod_{j \in m_{2}} \tilde{t}_{j}^{2}\right),
$$

where $V_{m_{1}}=\prod_{i<j} \sigma_{i j}$ for $i, j \in m_{1}$, and we proceed similarly for $V_{m_{2}}$. We therefore obtain a general formula for the amplitudes of 4D $\mathcal{N}=4$ Einstein-Maxwell theory:

$A_{n}^{\mathcal{N}=4}=\int d \mu^{4 \mathrm{D}} \frac{\operatorname{Pf} \mathcal{X}_{n_{2}}}{V_{n_{2}} V_{n}} T_{+}^{m_{1}} T_{-}^{m_{2}} R^{2}(\rho) R^{2}(\tilde{\rho}) \Omega_{F}^{\mathcal{N}=4}$,

where $\Omega_{F}^{\mathcal{N}=4}$ implements the $4 \mathrm{D} \mathcal{N}=4$ supersymmetry, arising as the reduction of $\Omega_{F}^{(2,0)}$, 


$$
\Omega_{F}^{\mathcal{N}=4}=\prod_{k=0}^{d} \delta^{2}\left(\sum_{i=1}^{n} t_{i} \sigma_{i}^{k} \eta_{i+}^{I}\right) \prod_{k=0}^{\tilde{d}} \delta^{2}\left(\sum_{i=1}^{n} \tilde{t}_{i} \sigma_{i}^{k} \eta_{i-}^{\hat{I}}\right) .
$$

The formula should be understood as summing over $d, \tilde{d}$ obeying $d+\tilde{d}=n-2$. However, it is clear from the superfields that we should require

$$
d=\frac{n_{2}}{2}+m_{1}-1, \quad \tilde{d}=\frac{n_{2}}{2}+m_{2}-1 ;
$$

recall that $n_{2}$ is even. Therefore, for a given number of photon and graviton multiplets, the summation over sectors becomes a sum over different $m_{1}, m_{2}$. We have checked (43) against many explicit amplitudes and have also verified that the integrand is identical to that of Ref. [12] for certain component amplitudes.

Discussion and conclusion.-In this Letter, we presented a formula for the tree-level $S$ matrix of 6D $(2,0)$ supergravity. The formula for single-flavor tensor multiplets was constructed via a SUSY reduction of the one for $(2,2)$ supergravity. We observed important simplifications in deriving the formula, particularly the appearance of the object $\operatorname{Pf} X_{n}$, crucially for the generalization to 21 flavors required for $(2,0)$ supergravity. By studying the soft limits of the formula, we were able to explore the moduli space of the theory. Via dimensional reduction, we also deduced a new formula for amplitudes of 4D $\mathcal{N}=4$ EinsteinMaxwell theory. Since 6D $(2,0)$ supergravity has a UV completion as a string theory, it would be of interest to extend our formula to include $\alpha^{\prime}$ corrections, perhaps along the lines of Ref. [26]. Also, a recent paper [27] introduces an alternative form of the scattering equations that treats even and odd points equally but uses a different formalism for supersymmetry. It will be interesting to study our formula in this formalism.

Our results provide an $S$ matrix confirmation of various properties of $(2,0)$ supergravity and the dimensionally reduced theory as predicted by string dualities. While the 10D theory has a dilaton that sets the coupling, in 6D this scalar is one of the 105 moduli fields and appears equally with the other 104 scalars. If one considers the compactification on $\mathrm{K} 3 \times \mathrm{T}^{2}$, standard $U$ dualities imply equivalence to the type IIA superstring theory on the same geometry or the heterotic string theory compactified to 4D on a torus. The formulas discussed in this Letter apply to all of these cases, at least at generic points of the moduli space.

We thank Nima Arkani-Hamed, Yvonne Geyer, and Shu-Heng Shao for the very helpful discussions. We also thank Freddy Cachazo, Alfredo Guevara, and Sebastian Mizera for discussions and correspondence on related topics. C. W. was supported by Royal Society University Research Fellowship No. UF160350. S.-Q.Z. was supported by Royal Society Grant No. RGFIR1\180037. M. H. would like to thank S. S. Gubser and Princeton University for their hospitality, and work done at Princeton was supported in part by the Department of Energy under Grant No. DE-FG02-91ER40671, and by the Simons Foundation, Grant No. 511167 (SSG). M. H. and J. H. S. were supported in part by the Walter Burke Institute for Theoretical Physics at Caltech and by U.S. DOE Grant No. DE-SC0011632.

[1] M. Heydeman, J. H. Schwarz, and C. Wen, M5-brane and D-brane scattering amplitudes, J. High Energy Phys. 12 (2017) 003.

[2] F. Cachazo, A. Guevara, M. Heydeman, S. Mizera, J. H. Schwarz, and C. Wen, The S matrix of 6D super Yang-Mills and maximal supergravity from rational maps, J. High Energy Phys. 09 (2018) 125.

[3] E. Witten, Perturbative gauge theory as a string theory in twistor space, Commun. Math. Phys. 252, 189 (2004).

[4] R. Roiban, M. Spradlin, and A. Volovich, Tree-level S matrix of Yang-Mills theory, Phys. Rev. D 70, 026009 (2004).

[5] F. Cachazo, S. He, and E. Y. Yuan, Scattering in three dimensions from rational maps, J. High Energy Phys. 10 (2013) 141.

[6] E. Witten, Some comments on string dynamics, arXiv:hepth/9507121.

[7] C. Cheung and D. O'Connell, Amplitudes and spinorhelicity in six dimensions, J. High Energy Phys. 07 (2009) 075.

[8] The anomaly cancellation has also been studied from the amplitude point of view, where one uses four-point amplitudes and unitarity; see Y.-t. Huang and D. McGady, Consistency Conditions for Gauge Theory $S$ Matrices from Requirements of Generalized Unitarity, Phys. Rev. Lett. 112, 241601 (2014); W. M. Chen, Y.-t. Huang, and D. A. McGady, Anomalies without an action, arXiv:1402.7062.

[9] The string-theory moduli space has singularities at fixed points of its $S O(5,21 ; \mathbb{Z})$ duality group. At such points, one or more tensor multiplets are replaced by non-Lagrangian $(2,0)$ conformal field theories, and a perturbative analysis is no longer possible. Therefore, the amplitudes presented in this Letter are applicable at generic points in the moduli space where we can treat the tensor multiplets as Abelian.

[10] N. Arkani-Hamed, F. Cachazo, and J. Kaplan, What is the simplest quantum field theory?, J. High Energy Phys. 09 (2010) 016.

[11] F. Cachazo, S. He, and E. Y. Yuan, Scattering of Massless Particles in Arbitrary Dimensions, Phys. Rev. Lett. 113, 171601 (2014).

[12] F. Cachazo, S. He, and E. Y. Yuan, Scattering equations and matrices: From Einstein to Yang-Mills, DBI and NLSM, J. High Energy Phys. 07 (2015) 149.

[13] H. Elvang, Y.-t. Huang, and C. Peng, On-shell superamplitudes in $\mathcal{N}<4$ SYM, J. High Energy Phys. 09 (2011) 031.

[14] For the superfield of the tensor, $\Phi(\eta)$, one may choose different $I, J$; however, only the case of $I=1, J=2$ leads to nonvanishing results when we integrate away $\tilde{\eta}$ 's from the amplitudes of $(2,2)$ supergravity. 
[15] The identity may be understood by studying the zeros and singularities on both sides of the equation. We have also checked it explicitly up to $n=12$.

[16] It should be emphasized that the artificial difference between the formulas of even- and odd-point amplitudes is due to the peculiar property of 6D scattering equations in the rational-map form, not specific to the chiral $(2,0)$ supergravity we consider in this Letter.

[17] Y. H. Lin, S. H. Shao, Y. Wang, and X. Yin, Supersymmetry constraints and string theory on K3, J. High Energy Phys. 12 (2015) 1.

[18] P. S. Aspinwall, K3 surfaces and string duality, arXiv:hepth/9611137.

[19] S. L. Adler, Consistency conditions on the strong interactions implied by a partially conserved axial vector current, Phys. Rev. 137, B1022 (1965).

[20] W. M. Chen, Y.-t. Huang, and C. Wen, From U(1) to $E_{8}$ : Soft theorems in supergravity amplitudes, J. High Energy Phys. 03 (2015) 150.

[21] A. Volovich, C. Wen, and M. Zlotnikov, Double soft theorems in gauge and string theories, J. High Energy Phys. 07 (2015) 095.

[22] For the study of the moduli space, the scalars we choose do not form a singlet; therefore, the most singular diagrams with a three-point amplitude on one side are not allowed. If instead the two scalars do form a singlet, then such diagrams dominate and contain a propagating soft graviton. The soft theorem for such a pair of scalars is simply given by Weinberg's soft graviton theorem attached with the threepoint amplitude, which we have also checked using our formula.

[23] Y.-t. Huang, Non-chiral $S$-matrix of $\mathcal{N}=4$ super YangMills, arXiv:1104.2021.

[24] As discussed in Ref. [2], the reduction to 4D kinematics may be so subtle in this formalism that only the middle sector of even $n$ (or next to the middle sector of odd $n$ ) is straightforward. The strategy we adapt here is that once we obtain the formula for the middle (or the next to middle) sector, we then generalize them for other sectors, which is straightforward.

[25] We do not consider $\Phi^{\hat{+} \hat{-}}$ here since they are identical to the vector, for which we have already included, as shown in Eq. (36).

[26] S. Mizera and G. Zhang, A string deformation of the ParkeTaylor factor, Phys. Rev. D 96, 066016 (2017).

[27] Y. Geyer and L. Mason, The polarized scattering equations for 6D superamplitudes, arXiv:1812.05548 [Phys. Rev. Lett. (to be published)]. 